East African Medical Journal Vol. 80 No. 2 February 2003

KNOWLEDGE, ATTITUDES AND PRACTICES OF PRIVATE MEDICAL PRACTITIONERS ON TUBERCULOSIS AMONG HIV/AIDS PATIENTS IN ELDORET, KENYA

S.O. Ayaya, MBChB, MMed, FDERM, Lecturer, Department of Child Health and Paediatrics, Moi University, P.O. Box 6121, Eldoret, Kenya, J. Sitienei, MBChB, Dip.(RIT) Medical Officer-in-Charge, National Leprosy and Tuberculosis Program, P.O. Box 5542, Eldoret, Kenya and W. Odero, MD, PhD, Associate Professor and Dean, Institute of Public Health, Moi University, P.O. Box 4606, Eldoret, Kenya, and J. Rotich, BSs, Senior Lecture, Department of Preventive Medicine and Epidemiology, Moi University, P.O. Box 4600, Eldoret, Kenya

Request for reprints to: Dr. S.O. Ayaya, Department of Child Health and Paediatrics, Faculty of Health Sciences, Moi University, P.O. Box 4600, Eldoret, Kenya

\title{
KNOWLEDGE, ATTITUDES, AND PRACTICES OF PRIVATE MEDICAL PRACTITIONERS ON TUBERCULOSIS AMONG HIV/AIDS PATIENTS IN ELDORET, KENYA
}

\author{
S. O. AYAYA, J. SITIENEI, W. ODERO and J. ROTICH
}

\begin{abstract}
Background: Tuberculosis (TB) is one of the major communicable diseases afflicting mankind today. Its prevalence is increasing with increase in HIV infection. It is important that doctors be able to correctly diagnose and institute proper management of patients with TB.

Objectives: To determine the knowledge, attitudes, and practices (KAP) of private medical practitioners in Eldoret on the management of TB.

Design: Cross-sectional descriptive qualitative study.

Setting: Private medical practitioners' clinics and the outpatient departments of private hospitals in Eldoret town, western, Kenya. Eldoret is a cosmopolitan town 350-km north west of Nairobi. It is the main town in the north Rift Valley with such infrastructure as roads, international airport, and banks.

Subjects: Private medical practitioners in Eldoret.

Results: Fifty three out of 70 private doctors were interviewed. Of these $84.9 \%$ were male. Only 5.7\% knew that sputum for AAFBs is collected on spot, early morning, and spot, whereas $69.8 \%$ and $13.2 \%$ said it should be collected on three and six consecutive early mornings respectively. Sputum and chest $X$-ray were the most common investigations used to diagnose TB. Few doctors knew that the clinical features considered as suspicious for TB in children were failure to thrive (FTT) $(\mathbf{2 0 . 6 \% )}$, contact with open TB case $(\mathbf{1 2 . 8 \%})$, and cough for more than two or more weeks $\mathbf{( 7 . 8 \% )}$. Others wrongly considered cough for four or more weeks $(9.2 \%)$. Features correctly considered of diagnostic value by a few of the private doctors in paediatric TB were: chest $X$ ray $(\mathbf{1 9 . 8 \%})$, FTT $(8.7 \%)$, positive sputum for AAFBs $(8.7 \%)$, and history of contact with TB case $(8.7 \%)$. A small number of doctors based their diagnosis on chest $X$ ray $(38 \%)$, AAFBs $(19 \%)$, and Keith-Jones criteria (6.3\%). There were 16 regimes mentioned and used for the treatment of TB. The NLTP recommended regimes such as 2RHZ/4RH, 2RHZE/6HE, 2RHZ/6HE and 2SHRZE/1RHZE/5HRE, were used by $9(19.6 \%), 2(4.3 \%), 0 \%$ and $0 \%$ of the doctors respectively. The rest used unrecommended regimes and no doctor used the re-treatment regime of 2SHRZE/1 RHZE/5RHE. Similar regimes were used for the HIV as for the non-HIV-infected patients. None of the interviewees had appropriate knowledge on all the areas of diagnosis, treatment, case recording, and follow up.

Conclusion: Most doctors were not aware of the correct diagnosis and treatment of TB and many used unrecommended treatment regimes. They were generally unfamiliar with the recording system of TB cases. Most doctors did not know the definitions of the various re-treatment cases. Continuing medical education on clinical management of TB patients is needed for doctors in private practice.
\end{abstract}

\section{INTRODUCTION}

Tuberculosis is an infectious disease, which is prevalent in conditions of poverty, low socio-economic status and high HIV infection(1). It was first recorded in Africa in 1897 by Albert Cook(2) This disease was on the decline from the 1950's to the early 1980's. However since the mid 80's with the advent of HIV/ AIDS the prevalence of TB has been increasing especially in sub-Saharan Africa. The incidence of tuberculosis among HIV infected patients is $45 \%$ and about $50 \%$ of HIV infected patients will develop TB 
in their lifetime(3). With increasing incidence of HIV infection, TB is also increasing and therefore private medical practitioners are seeing more TB patients. In Eldoret as is common in other Kenyan and African cities, many doctors manage cases outside their areas of specialization. For the proper management of TB, the physician needs to have a high index of suspicion to be able to take appropriate history, order investigations, make the correct diagnosis and decide to either institute treatment or refer the patient promptly. The diagnosis of TB is based on good history, clinical examination, and laboratory investigations. These require good knowledge to institute. Where doctors have had a low index of suspicion and poor knowledge a lot of patients have not been investigated properly, some have been put on inappropriate and unrecommended regimes and many TB cases have been diagnosed at postmortem(4,5). It has been reported that private doctors tend not to refer TB patients appropriately for proper treatment and follow up(6). It was therefore important to find out how these private doctors managed the TB cases.

The aim of this study therefore was to examine the knowledge, attitudes, and practices of private doctors. This would reveal the gaps in their knowledge and practice on the management of TB. Thus what needs to be covered in continued medical education sessions on TB would be identified.

\section{MATERIALS AND METHODS}

Study area: The study was conducted in Eldoret town, which is $350 \mathrm{Km}$ northwest of Nairobi. It is a cosmopolitan town in the highlands of the North Rift Valley of Kenya and the headquarters of the Uasin-Gishu District. There is an estimated population of 250000 . Most people in the district depend on farming. Eldoret houses the Moi Teaching and Referral Hospital (MTRH) that serves the North Rift. Valley, Western and Nyanza provinces. There are four private, two public hospitals, several municipal health centers and private medical clinics in the town.

Study population: The study population included medical doctors practicing privately either full or part time.

Inclusion criteria: All doctors who were in private practice fully, partially, or on locum were interviewed. Upto three attempts were made to interview the doctors, those found and consented to an interview were included.

Exclusion criteria: Doctors in Eldoret town who were not involved in some form of private practice, those who could not give consent or keep appointments on three occasions as well as those out of the town were excluded. study.

Study design: This was a cross-sectional descriptive

Study tool: The study tool was a standard questionnaire with questions which included the respondent's age, sex, speciality, and KAP on tuberculosis management.

Sampling methods and size: Purposive sampling was used to identify and interview the doctors in private practice either in their offices or in the hospitals.

Data collection: The data was collected through a face to face interview conducted by the researchers (ASO and JS). Each interview took about thirty minutes and the entire survey took about six months. The knowledge questions were put to the respondents without prompting them. The answers were recorded as given and later the number and percentage of respondents that had given an answer was worked out whether the answer was correct or wrong. The attitudes were statements made by the interviewer and the respondent requested to state whether he/she disagreed completely, agreed partially, agreed completely or did not know what to say. The respondents were also asked various practice questions and their responses recorded as given. The knowledge and practice answers were compared with the expected answers for their appropriateness and commented on.

Data management and analysis: The data collected was entered in a computer on the SPSS program (Statistical Package for Social Sciences). Descriptive statistics were computed. The knowledge was scored, the mean scores compared among the various specialists, the t-test was used to determine the significance of the differences. The analysis of attitudes employed the Lickert scale of 0 to 3 as follows: disagree completely scored 3 , agreed partially 2 , agreed completely 1 , and do not know 0 .

Ethical considerations: Approval to conduct the study was obtained from the institutional research and ethics committee (IREC) of the Faculty of Health Sciences, Moi University. A verbal consent for interview was obtained from each of the doctors after explaining the purpose of the study. Neither the names nor the addresses of the respondents were recorded and the respondents were assured of confidentiality.

\section{RESULTS}

Demographic data: Out of 70 private medical practitioners, $53(75.7 \%)$ were interviewed. Among them, there were four $(7.5 \%)$ paediatricians, four $(7.5 \%)$ physicians, eight (15.1\%) obstetricians/gynaecologists, nine $(17.0 \%)$ surgeons, one (1.9\%) psychiatrist, 23 (43.4\%) general practitioners and four $(7.5 \%)$ other specialists. There were $45(84.9 \%)$ males. The age ranged from 27 to 66 years with a mean of $43 \pm 10.5 \mathrm{SD}$.

Knowledge on the diagnosis of TB: The correct method of sputum collection was given as: Early morning for three consecutive days 37(69.8\%), early morning for six consecutive days seven $(13.2 \%)$, on first contact with the patient, early following morning, and spot during second contact with patient three $(5.7 \%)$ and other six (11.4\%), Thus only $5.7 \%$ of the doctors knew the correct method of collecting sputum.

When the doctors were asked about the tests that the sputum could be subjected to make a diagnosis of $\mathrm{TB}$, the following were their responses Ziel-Nielsen (ZN) stain for acid alcohol fast bacilli (AFBs) 42 (42.9\%), culture and sensitivity $32(32.7 \%)$, acid-fast bacilli (AFBs) eight $(8.2 \%)$, and five others $(5.1 \%)$. Among the others were tests such as the PCR, which is not yet routinely used, but the respondents who included 
University lecturers were unaware of this. Lecturers are expected to be aware of even experimental tests for the purpose of teaching and enlightening others.

The interviewes were asked to name tests other than those performed on sputum that could be used in the diagnosis of TB and the following were their answers: Chest X-ray 24(15.5\%), X-ray of other affected organs 24(15.5\%), biopsy 22(14.2\%), ESR 22(14.2\%), haemogram 19(12.3\%), Mantoux test 14(9\%) and others $29(18.7 \%)$.

The doctors were asked if they had heard of KeithJones criteria, how many parameters were on it, and the interpretation of the scores and the results were: $22(41.5 \%)$ had heard, only one $(1.9 \%)$ doctor knew that there were 13 parameters, the rest $52(98.9 \%)$ did not know. Of the 22 doctors who had heard of the criteria, only three (13.6\%) interpreted the scores correctly.

The doctors were asked how many positive sputum smears were required to define a smear positive case and they said: one, two or three positive sputum in 35 $(67.3 \%), 5(9.6 \%), 9(173 \%)$ of the cases respectively. Three $(5.3 \%)$ of the doctors did not know the definition. Therefore only $9.6 \%$ knew that two positive smears were required to define a smear positive case.

Knowledge about TB treatment: The doctors were asked to list the individual drugs used in treatment of TB and the following drugs were listed in these proportions: ethambutol $44(20.6 \%)$, isoniazid 43 $(20.1 \%)$, rifampicin $41(19.2 \%)$, streptomycin 29 (13.6\%), pyrazinamide $25(11.7 \%)$, thiacetazone $21(10.7 \%)$, quinolones six $(2.8 \%)$ and others three (1.4\%).

When asked to give the average daily dosages of the anti tuberculous drugs listed, there were 212 responses. Of these four $(1.9 \%)$ were correct $18(8.5 \%)$ were wrong and $193(97 \%)$ did not know the dosages.

Relapse was defined correctly as "patients who become smear positive after receiving treatment for TB to completion and being declared cured" by $30(56.6 \%)$, wrongly by $12(22.6 \%)$ and $11(20.8 \%)$ did not know.

Treatment after failure was defined correctly as "patients who on initial treatment for smear positive pulmonary TB remained or became again smear positive at five months or later during the course of treatment by nine (17\%), wrongly by $20(37.7 \%)$ and $24(45.3 \%)$ did not know.

Treatment after default was defined wrongly by $29(54.7 \%)$ and $24(45.3 \%)$ did not know. None of the respondents knew that the definition of treatment after default was "patients who return to treatment after leaving treatment for more than two months".

Chronic case was defined wrongly by $19(35.8 \%)$ and $34(64.2 \%)$ did not know. None of the doctors knew that the correct definition was "patients who continue to be smear positive after completion of fully observed treatment regime."
When asked what the acronym DOTS stood for: $27(50.9 \%)$ did not know, 19(35.8\%) said that it was directly observed treatment, $4(7.5 \%)$ knew that it was directly observed treatment short course chemotherapy and others three $(5.71 \%)$.

Practices: Patients were managed by the attending doctor $26(55.3 \%)$, referred to; NILTP 12 (25.5\%), physician $9(19.1 \%)$. No patient was referred to paediatricians.

The doctors were asked to say how they had made a diagnosis of TB in the last patient they had seen and the following methods were given; Chest X-ray 30 (38\%), positive sputum for AAFBs $15(19 \%)$, clinical $14(17.7 \%)$, Keith-Jones criteria five (6.3\%), histology (AAFBs) five (6.3\%), fine needle aspirate (AAFRs) two $(2.5 \%)$ and others eight $(10.1 \%)$.

Smear positive $10(22.2 \%)$, smear negative seven $(15.6 \%)$, extra-pulmonary four $(8.9 \%), 10(22.2 \%) \mathrm{did}$ not know how to record and $14(31.1 \%)$ recorded them wrongly.

The number of positive smears that were relied on to make the diagnosis of smear positive cases were: one $16(57.1 \%)$, two, 3(10.7\%) and three 9(32.1\%).

The sputum was collected from patients as follows: six early morning on consequtive days $4(10.5 \%), 3$ early mornings on consecutive days $32(60.7 \%)$, spot early morning and spot $3(7.9 \%),(13.2 \%)$ did not collect sputum while, (15.8\%) used other methods.

The most common side effect was dermatitis. Side effects like red urine, optic neuritis, and nephrotoxicity were mentioned as possible but were not observed by any doctor.

Only one $(3.3 \%)$ doctor noted some cases of drug resistance, $24(71.7 \%$ ) did not observe any case of drug resistance while $8(24.2 \%)$ did not know if there were any. Twenty six $(56.5 \%)$ of the doctors referred the patients to the NLTP for registration. Twenty $(57.1 \%)$ of the doctors said that their patients were able to access free drugs from the NLTP, 8(22.9\%) said they were unable and $7(20 \%)$ did not know whether they got the free drugs.

Table 1 shows the factors that the doctors felt would raise the suspicion of TB in a child or an adult. Very few doctors knew that failure to thrive contact with an open TB case, and cough for 2 or more weeks could raise suspicion of TB in a child. Very few also knew that in an adult, TB should be suspected if there was history of cough for 3 or more weeks, weight loss, and contact with an open TB case.

Table 2 shows the important features in the formulation of a diagnosis of $\mathrm{TB}$ in children. Even though the chest X-ray, failure to thrive, demonstration of acid alcohol fast bacilli (AAFBs) in sputum or any other tissue, contact with open case of TB, positive Mountoux (tuberculin) test and cough for 2 or more weeks are used in the formulation of a diagnosis of TB in children, less than $20 \%$ of the doctors knew these facts. Thus more than $80 \%$ did not know. 
Table 3

Table 3 demonstrates the parameters on the KeithJones criteria that the interviewed doctors knew. Only 7 out of 13 parameters were mentioned. It also shows how many knew the correct score for the parameters they had mentioned. Thus correct meant the given for the parameter was right, wrong meant the score given for the parameter was not correct while do not know meant that the respondent did not know the score given for the parameter they had mentioned.

Table 1

Factors that would make one suspect TB in a patient

\begin{tabular}{lcc}
\hline Factor & $\begin{array}{c}\text { Child } \\
\text { Freq }(\%)\end{array}$ & $\begin{array}{c}\text { Adult } \\
\text { Freq }(\%)\end{array}$ \\
\hline $\begin{array}{l}\text { Failure to thrive (FTT) } \\
\text { Contact with an open TB }\end{array}$ & $29(20.6)$ & - \\
$\quad$ case & $18(12.8)$ & $6(4.2)$ \\
Cough lasting 4 or more weeks & $13(9.2)$ & $18(12.5)$ \\
Weight loss & $13(9.2)$ & $30(20.8)$ \\
Cough lasting 2 or more weeks & $11(7.8)$ & $13(9.0)$ \\
$\begin{array}{l}\text { Fever } \\
\text { Lack of response to }\end{array}$ & $11(7.8)$ & 0 \\
$\quad$ antibiotics & $8(5.7)$ & $9(6.3)$ \\
Lymphadenopathy & $5(3.5)$ & 0 \\
Haemoptysis & 0 & $10(6.9)$ \\
Cough lasting more than & & \\
$\quad 3$ weeks & 0 & $9(6.3)$ \\
Others & $33(23.4)$ & $24(16.7)$ \\
Total & $141(100)$ & $144(100)$ \\
\hline
\end{tabular}

Table 4 indicates the fixed drug combinations (FDCs) that the doctors knew. They were asked to say what was contained in the FDCs. Correct meant the combination given was right, wrong meant it was incorrect and do not know meant they did not know the drugs contained in the FDCs they had listed.

Table 2

Diagnostic features of $T B$ in children

Feature

Frequency (\%)

\section{C-Xray}

$25(19.8)$

Failure to thrive(FTT)

$11(8.7)$

Alcohol acid fast bacilli (AAFB)

$11(8.7)$

History of contact with an open case of TB 10 (7.9)

Weight loss

Raised ESR

$8(6.3)$

$7(5.6)$

Fever

Positive Mantoux test

Cough lasting more than 2 weeks

Cough lasting more than 4 weeks

Haemogram

Others
Parameters on the Keith-Jones criteria with their scores

\begin{tabular}{lcccc}
\hline Parameter & \multicolumn{3}{c}{ Scores } & \\
& Correct & Wrong & Do not & Total \\
\hline Contact with PTB case & 1 & 21 & 5 & 27 \\
Positive mantoux & 2 & 17 & 6 & 25 \\
AAFB positive & 4 & 10 & 2 & 16 \\
Suggestive C-Xray & 6 & 5 & 5 & 16 \\
Present BCG scar & 4 & 6 & 5 & 15 \\
Non-specific S/S & 0 & 1 & 1 & 2 \\
Age $\leq$ 2 years & 1 & - & - & 1 \\
\hline Total & 18 & 60 & 24 & 102 \\
\hline
\end{tabular}

Table 4

Knowledge on fixed drug combinations

\begin{tabular}{lcccc}
\hline FDC & Correct(\%) & Wrong(\%) & $\begin{array}{c}\text { Don't } \\
\text { Know(\%) }\end{array}$ & Total(\%) \\
\hline Rifater & $14(42)$ & $11(33)$ & $8(24)$ & 33 \\
Rifinah & $14(42)$ & $9(27)$ & $10(30)$ & 33 \\
AKT4 & $4(12.5)$ & $12(37.5)$ & $16(50)$ & 32 \\
Thiazide & $2(13.3)$ & $2(13.3)$ & $11(73.6)$ & 15 \\
Ethizide & $2(13.3)$ & $2(13.3)$ & $11(73.6)$ & 15 \\
Total & $36(28.1)$ & $36(28.1)$ & $60(46.9)$ & $128(100)$ \\
\hline
\end{tabular}

Table 5 shows the average scores attained by the various cadres of doctors on each knowledge question. It also demonstrates the expected possible maximum score for each question. All the cadres of doctors scored very low compared to the maximum possible score. Though pediatricians scored the highest mean scores for most of the questions, the mean score was still very low. For example, their best score was on K7 where they had a mean of 17 out of $30(56.6 \%)$ but their worst score was on kI6 where their mean was 1.3 out of 30 $(4.3 \%)$. Thus all the different specialists interviewed had very low mean scores on all the questions.

Table 5

Mean scores of knowledge of TB management

\begin{tabular}{lccccccc}
\hline Specialists & \multicolumn{7}{c}{ Knowledge } \\
\cline { 2 - 8 } & K4 & K7 & K8 & K9 & K12 & K16 & K18 \\
\hline 1 & 2 & 17 & 14.3 & 14.8 & 8.8 & 1.3 & 4.8 \\
2 & 1.7 & 6 & 10.7 & 7.3 & 0.0 & 0.0 & 3.3 \\
3 & 1.6 & 9 & 12.0 & 8.3 & 0.0 & 0.0 & 0.3 \\
4 & 2.4 & 8.9 & 10.8 & 12.4 & 0.0 & 1.3 & 1.5 \\
5 & 2 & 14 & 14.0 & 7.0 & 0.0 & 0.0 & 0.0 \\
6 & 2 & 12.6 & 12.6 & 11.6 & 3.4 & 0.2 & 3.2 \\
7 & 2 & 10.3 & 8.1 & 9.3 & 0.0 & 0.7 & 1.4 \\
Mps & 5 & 30 & 30 & 30 & 20 & 30 & 20 \\
\hline
\end{tabular}


Key:

1 Paediatricians

2 Physicians

3 Obstetricians/gynaecologists

4 General surgeons

5 Psychiatrists

6 General practitioners

7 Others

K4 correct method of collecting a specimen of sputum from a patient for AAFBs

K7 what would make one suspect TB in a child?

K8 what would make one suspect TB in an adult?

K9 diagnostic features of TB in children

K12 interpretation of the Keith-Jones scores

K16 daily average doses of the various individual antituberculous drugs

K18 current regimes used in the treatment of tuberculosis. Mps maximum possible score.

Table 6

Attitudes towards TB patients

\begin{tabular}{lccccc}
\hline Attitude & $\begin{array}{c}\text { Disagree } \\
\text { completely } \\
(\%)\end{array}$ & $\begin{array}{c}\text { Agree } \\
\text { partially } \\
(\%)\end{array}$ & $\begin{array}{c}\text { Agree } \\
\text { completely } \\
(\%)\end{array}$ & $\begin{array}{c}\text { Do not } \\
\text { know } \\
(\%)\end{array}$ & Total \\
\hline 24 & $12(22.6)$ & $8(15.1)$ & $32(60.4)$ & $1(1.9)$ & $53(100)$ \\
25 & $21(39.6)$ & $5(9.4)$ & $26(49.1)$ & $1(1.9)$ & $53(100)$ \\
26 & $10(18.9)$ & $7(13.2)$ & $33(62.3)$ & $3(5.7)$ & $53(100)$ \\
27 & $14(58.3)$ & $2(8.3)$ & $2(8.3)$ & $6(25)$ & $24(100)$ \\
29 & $37(82.2)$ & $3(6.7)$ & $4(8.9)$ & $1(2.2)$ & $45(100)$ \\
\hline
\end{tabular}

Key:

24 most TB patients in Kenya have HIV/AIDS

25 HIV/AIDS patients deserve special regimes for the treatment of TB

26 HIV/AIDS patients with TB respond differently to treatment

27 Keith-Jones criteria are of no use in the diagnosis of TB in children with HIV/AIDS.

29 DOTS is not feasible in the developing world

29 management of TB should be left to NLTP staff

Table 7 shows the anti-TB regimes listed by the doctors and compares this with the regimes they used in treating TB patients who were infected and not infected by the HIV virus. It demonstrates that the drugs known by the doctors were the same ones used for both types of patients. The HIV infected TB patients were not put on any different regimes
Table 7

Knowledge and practice on the regimes used in the treatment of Tuberculosis in HIV and non-HIV infected patients

\begin{tabular}{llll}
\hline Regime & \multicolumn{2}{l}{ Knowledge(\%) } & Practice \\
& & Non-HIV(\%) & HIV(\%) \\
\hline 1STH/17TH & $2(3)$ & 0 & 0 \\
1STH/11TH & $2(3)$ & $1(3.8)$ & $1(5)$ \\
2RHZ/6RH & 0 & $2(7.7)$ & $1(5)$ \\
2RHZ/6EH & $3(4.5)$ & 0 & 0 \\
2RHZ/6EH & $3(4.5)$ & $1(3.8)$ & $1(5)$ \\
2RHZE/4EH & $7(10.4)$ & $4(15.4)$ & $5(25)$ \\
2RHZE/4EH & $2(3)$ & $1(3.8)$ & $1(5)$ \\
2RHZ/6EH & $2(3)$ & $1(3.8)$ & $1(5)$ \\
ER/ER & $1(1.5)$ & $1(3.8)$ & $1(5)$ \\
2RHZE/6RHZ & $4(6)$ & $2(7.7)$ & $1(5)$ \\
2RHZE/6RH & $2(3)$ & $1(3.8)$ & $1(5)$ \\
2RHZ/6E & $1(1.5)$ & $1(3.8)$ & $1(5)$ \\
2SRHZ/4RH & $3(4.5)$ & $4(15.4)$ & $1(5)$ \\
6RHZ & $4(6)$ & $2(7.7)$ & $4(20)$ \\
2SRHZ/6RHZ & 0 & $2(7.7)$ & $1(5)$ \\
2RHZE/4RH & 0 & $2(7.7)$ & 0 \\
Do not know & $18(26.9)$ & 0 & 0 \\
Others & $13(19.4)$ & $1(3.8)$ & \\
\hline Total & $67(100)$ & $26(100)$ & $20(100)$ \\
\hline
\end{tabular}

NB: Some respondents mentioned more than one regime.

Table 8 demonstrates the side effects of the antituberculosis drugs that the interviewed doctors knew as compared to the ones they observed in the patients they were treating. The doctors knew more side effects than they observed probably because of lack of follow up or they did not look out for them or ask about them.

Table 8

Knowledge and practice on the side efflects of the anti$T B$ drugs

\begin{tabular}{lll}
\hline Side Effect & Knowledge(\%) & Practice(\%) \\
\hline Dermatitis & $20(18.2)$ & $5(12.8)$ \\
Hepatotoxicity & $20(18.2)$ & $6(15.4)$ \\
Ototoxicity & $16(14.5)$ & 0 \\
None (do not know) & $15(13.6)$ & $22(56.4)$ \\
Peripheral neuritis & $14(12.7)$ & $1(2.6)$ \\
Red urine & $9(8.2)$ & 0 \\
Optic neuritis & $7(6.4)$ & 0 \\
Nephrotoxicity & $6(5.5)$ & 0 \\
Others(GIT) & $3(2.7)$ & $5(12.8)$ \\
\hline Total & $110(100)$ & $39(100)$ \\
\hline
\end{tabular}




\section{DISCUSSION}

The diagnosis of tuberculosis is normally based on the history, clinical examination, and laboratory investigations. An adult patient is considered a TB suspect if he has coughed for three or more weeks, and/ or he has haemoptysis, shortness of breath, chestpain, loss of appetite, loss of weight, night sweats and fever(1) A child is suspected of having TB if he has been coughing for two or more weeks, has been in contact with a TB case, and has failure to thrive(1). Most of the interviewees did not know this. This indicated a low index of suspicion that could most likely result in wrong diagnosis, delayed investigations and diagnosis leading to either wrong or delayed treatment with the attendant complications. The gold standard in the diagnosis of TB is the demonstration of acid alcohol fast bacilli (AAFBs) in sputum or gastric lavage specimens using the ZielNielsen (ZN) stain(1). The other routine investigations that assist in making the diagnosis include chest X-ray, Mantoux test, and haemogram. There are more rapid and convenient tests for serodiagnosis and culture, which can be done on specimens other than sputum of which most of our doctors were unaware(9-18). The serodiagnostic tests are faster than sputum examination for AAFBs and can be used in children who cannot produce sputum and in extrapulmonary TB(19-20). Though the tests are not available for routine use it was expected that some of the doctors especially consultants and lecturers would be aware of them for academic reasons. The correct method of collecting sputum for $\mathrm{ZN}$ stain is: first specimen collected at the first interview on the spot, second specimen the patient is given a sputum mug and asked to put the following early morning specimen before the second interview and the third specimen is collected on the spot during the second interview(7). Only a few doctors knew this. Most of them collected three consecutive early morning specimens leading to delay in diagnosis and possibly loss of patients. However, though most doctors knew of sputum for AAFB, and culture and sensitivity only two $(6.8 \%)$ were aware of the PCR and ELISA methods $(18,21)$. The chest X-ray was the investigation most commonly used for the diagnosis of TB probably due to its availability and affordability. The Mantoux test is difficult to interpret, less sensitive than the BCG and is not readily available in most health facilities. This could explain why no doctor had used it. No doctor mentioned BCG as a possible method of investigation(22). The diagnosis of tuberculosis in children is quite difficult(1). Only a very small proportion of children have tuberculosis that is smear positive and many children cannot produce sputum therefore points of importance in determining a diagnosis in children, in order of priority are; a history of contact with a case of infectious tuberculosis, especially in the same household, chest X-ray showing unilateral lymphadenopathy and/or shadows in the lung fields indicating infiltration and a positive tuberculin skin test, where it is available. The Keith Jones criterion, which has 13 parameters improves the chances of diagnosing TB in children(23). The Keith Jones criteria were unfamiliar to most of the doctors interviewed. Only $8.5 \%$ of the responses were correct scores for the named parameters. This explained why only $6.3 \%$ had used this criteria to make a diagnosis of TB in their patients. The foregoing shows that most doctors had a low index of suspicion, did not know the diagnostic features of TB in children, and could not use the Keith-Jones criteria. This may have been due to complacence from the fact that this disease was almost eradicated and so not enough emphasis was put on it in the medical schools and continued medical education sessions. However, with the re-emergence of TB alongside HIV infection, the management of the disease should be emphasized in the medical schools and during CMEs for practicing doctors. This should apply to most developing countries. The Keith-Jones criteria are no longer in use in developed countries like the USA because of a low prevalence of TB and availability of other diagnostic tools. In Kenya and other developing countries, where resources are scarce, its use will help improve the diagnostic ability and timely institution of appropriate treatment. The paediatricians had higher mean scores on the diagnosis of TB in children than the other doctors. However, there was no doctor who had referred his or her patients to a paediatrician. The doctors should be encouraged to refer children to the paediatricians.

An effective anti-TB regime must contain at least two bactericidal drugs and be given for long enough to avoid relapse(24). The first line drugs are streptomycin, isoniazid, rifampicin, ethambutol, pyrazinamide, and thiacetazone. The second line drugs are the fluoroquinolones like ciprofloxacin, levofloxacin and sparfloxacilin among others. The interviewed doctors were familiar with the first line drugs but not the second line. Their mean scores on the daily dosages of the first line drugs were very low. Adequate chemotherapy requires appropriate combination and corrects dosage prescription to avoid resistance(1). The World Health Organization recommends the use of fixed drug combinations (FDCs) for these and other reasons(24). Various FDCs (RHZ, RH and EH) are used in by the Kenyan NLTP as per WHO and IUATLD guidelines. The regimes currently recommended by the NLTP of Kenya are: 2ERHZ/6EH for smear positive and severely ill, 2RHZ/6EH for sputum negative and extra-pulmonary (not severely ill), 2SHRZE/1RHZE/5RHE for patients on re-treatment, $2 \mathrm{RHZ} / 4 \mathrm{RH}$ for children younger than 15 years. (The numbers signify the duration of treatment in months)(7). The recommended regimes were only familiar to about $10 \%$ of the doctors. No doctor mentioned the re-treatment regime and none had put a patient on it. This may have been due to the fact that they could not define most re-treatment cases nor did they know the re-treatment regimes. Some doctors did not know about intensive and continuation phases 
and therefore put their patients on six months of RHZ. In practice, the regimes used for HIV negative patients were similar to those used for HIV positive patients, which was good.

Patients who are smear positive and have been treated for a month or more previously should be put on re-treatment. These are classified as relapses, treatment after failure, and treatment after default. Relapses are patients who become smear positive after receiving treatment for $\mathrm{TB}$ to completion and being declared cured. Treatment after failure are patients who on initial treatment for smear positive pulmonary TB remained or became again smear positive at five months or later during the course of treatment. Treatment after default is patients who return to treatment after leaving treatment for more than two months. Chronic cases are those who continue to be smear positive after completion of a fully observed treatment regime. A lot of the doctors interviewed knew the correct definition of relapse and treatment after failure. None knew the definition of treatment after default and chronic cases. In practice only one doctor noted a case of drug resistance but he did not put the patient on re-treatment regime probably due to lack of knowledge. Only $7.5 \%$ knew the meaning of the acronym DOTS, implying that they probably did not practice it. Since the doctors did not know this acronym, it is unlikely that they knew the five elements of DOTS(25) Though the doctors were familiar with seven side effects of the drugs used, they hardly observed them because of not following and monitoring the patients' side effects. These drugs have various side effects that the doctors should be aware of and advice their patients accordingly.

Any person treated for tuberculosis should be reported as a case. Those who have AAFBs in sputum should be recorded as smear positive, the others are either smear negative or extra-pulmonary. One requires 2 positive sputa to make a diagnosis of a smear positive case(1). Less than $10 \%$ of the doctors knew this while the majority said one positive sputum. In practice most had relied on one positive sputum to make the diagnosis of smear positive case. Considering that most doctors did not know how to record their cases and did not know the definition of the re-treatment cases, it would be difficult to interpret their records and use them for reporting and epidemiological purposes.

Though the doctors had the attitude that most TB patients had HIV infection and that those infected with HIV required special regimes, these did not influence their practice. They did not put these patients on any special regimes. This was good although it may have been due to lack of knowledge of any other regimes to use. They were also willing to participate in the management of these patients and did not hold the notion that this was the problem of the NLTP staff. The NLTP should therefore update the practicing physicians on the current trends in the management of TB.
Multi-drug resistance (MDR) is likely to occur if there is: lack of compliance, or when monotherapy, inadequate dosages, and inappropriate regimes are used(24). Our findings of low index of suspicion, lack of diagnostic, and treatment knowledge are a recipe for creating multidrug resistance and cases for retreatment. This could result in a large cohort of patients with drug resistant organisms. It would be very difficult to identify these considering that the doctors do not keep proper records and they monitor their patients poorly. Similar findings were observed in Nairobi where two thirds of the data was missing from the register in a retrospective study in an NLTP run clinic(25). We believe that this situation is replicated in other parts of Kenya, Africa, and other developing countries. Although we studied private doctors, similar findings are likely to be found among public sector doctors without any private practice nurses and clinical officers in private practice. TB in Kenya is stigmatized as it is elsewhere. Therefore patients who can afford prefer to be treated in private clinics and hospitals and they do not like to attend public clinics and hospitals where the medical and clinical officers trained in the management of TB work.

In conclusion most of the interviewed private medical practitioners had low index of suspicion of TB in adults and children, did not know the appropriate method for sputum collection and were unfamiliar with the current anti-TB regimes. Most of them used inappropriate and unrecommended regimes to treat tuberculosis and were unfamiliar with the system of recording TB cases. A lot of the doctors did not know the definition of the various re-treatment cases. Most doctors had good attitudes towards the disease. patients, and the Kenyan NLTP.

We recommend that the Ministry of health through the medical officer's in-charge of NLTP all over the country should appraise the practicing private physicians on current management of tuberculosis. The teaching of TB should be emphasized in the undergraduate and postgraduate medical curricula in Kenya and Africa. Doctors both in private and public practice should endeavour to appraise themselves on changes in the management of TB. The KAP on management of TB among the other cadres of paramedical staff who also treat TB patients privately should be established.

\section{ACKNOWLEDGEMENTS}

To the Dean of the faculty of Health Sciences, Moi University for the co-operation and conducive environment that made it possible to do this work, the doctors who spared their time to respond to the questions, our families for bearing with us during the study period and anyone else that directly or indirectly positively contributed to this work. 


\section{REFERENCES}

1. Management of tuberculosis: A guide for low-income countries 5th edition, edited by: Donald A., Einarson, Hans L. Riecher, Thurinder Annadottir, Arnomed, Trebuccl. Paris. 2000.

2. Daniel, T.M. The early history of tuberculosis in East Africa: Insights from the records of the first twenty years of Mengo hospital and review of the literature. Interna. $J$. Tubercul. and lung dis. 1998; 2:784-790.

3. Okot-Nwang, M., Wabwire-Mageni, F. and Kagezi, VB. Increasing prevalence of tuberculosis among Mulago hospital admissions, Kampala, Uganda. (1985-1989) Tubercle and lung dis. 1993; 74:121-125.

4. DeRiener, K, Rudoy, I., Schecter, F., Hopewell, G.F., and Daley, C.L. The epidemiology of tuberculosis diagnosed after death in San.Fransisco 1986-1995. Interna. J. Tubercu. lung dis. 1999; 3:498-493.

5. Johanson, E., Divan, V.K., Huong, N.D. and Abberg, B.M. Staff and patients attitude to tuberculosis and compliance with treatment: an exploratory study in a district in Vietnam. Tubercle and lung dis. 1996; 77:178-183.

6. Kenya National leprosy and tuberculosis Program (NLTP). Beyers, N., Gie, P.P., Schaaf, H.S., Van, Zyl, S., Talent, J.M., Nel, E.D. and Donald, P.R.

7. A prospective evaluation of children under the age of 5 years living in the same household as adults with recently diagnosed pulmonary tuberculosis. Interna. J. Tuberc. and lung dis. 1997; 1:38-43.

8. Schaaf, H.S., Nel, E.D., Beyers, N., Gue, R.P. and Donald, P.R. A decade of experience with mycobacterium tuberculosis culture from children: a seasonal influence on incidence of childhood Tuberculosis. Tubercle and Lung dis. 1996; 77:43-46.

9. Hoffner, S.E., Norberg, R., Carlos Toro, J, Winquist, N., Koivula,T., Dias, F., Svenson, S.B. and Kalleins, G. Direct detection of $M$. tuberculosis in sputum samples from Guinea Bissau by an RNA target - amplified test system. Tubercle and lung Dis. 1996; 77: 67-70.

10. Gupta, S., Kumar, S., Bamralikar, J.N., and Gupta, S.K. Diagnostic utility of the estimation of mycobacterium antigen 60 Specific immunoglobulins: $\operatorname{IgM}, \operatorname{IgA}$, and $\operatorname{IgG}$ in the sera of cases of adult human tuberculosis. Tubercle and lung dis. 1995; 76:418-424.

11. Mustaafa A.S., Ahmed, A., Abal, A,T. and Chang, T.D. Establishment and evaluation of a multiple polymerase chain reaction for detection of mycobacterium and specific identification of $\mathrm{M}$. tuberculosis complex. Tubercle and lung disease. 1995; 76:336-343.

12. Daleine, G. and Langerange, P.H. Preliminary evaluation of a Mycobacterium tuberculosis lipo-oligosaccharide (LOS) antigen in the serological diagnosis of tuberculosis in HIV seropositive and seronegative patients. Tubercle and lung disease. 1995; 76:234-239.

13. Thybo, S., Richter, C., Wachmann, H., Marselle, S.Y., Mtoni, I. and Anderson, A.B. Humoral response to M. tuberculosis specific antigens in African Tuberculosis patients with high prevalence of HIV infection. Tubercle and lung disease. 1995; 76:149-155.

14, Gleunon, M., Smith, T, Cornica, M, et al. The ribosomal intergenic spacer regime: a target for the PCR based diagnosis of tuberculosis. Tubercle and lung disease. 1994; 75: 353-356.

15 Soni, G.R., O’Brien, R.J., Mfinanges, G.S. and Ipuge, Y.A. Evaluation of the mycodot test in-patients with suspected tuberculosis In Tanzania. Interna. J. Tubercul. and lung dis. 1999; 3: 231-238.

16. Khomentho, A.G., Bayenshy, A.V., Chernusova, L.N. et al . Serodiagraphs of tuberculosis: detection of mycobacteriosis antibodies and antigens. Tubercle and Ling Dis. 1996; 77: 510-515.

17. Streaton, J.A., Desen, N. and Jones, S.L. Sensitivity and specificity of gamma interferon blood test for tuberculosis infection. Interna. J. Tuberculo. and lung dise.. 1998; 2: 443-450.

18. Gupta, I., Jani, A., Singh, N.B., Chatuvedi, V. and Aggarwal, $\mathrm{K}$. Role of antigen specific circulating immune complexes in the diagnosis of tuberculosis. International journal of tuberculosis and lung dise. 1998; 2:456-461.

19. Rathan, A., Gupta, S.K., Singh, S., et al, D. Detection of antigens of M. tuberculosis in patients of infertility by Monoclonal antibody based enzyme linked immunosorbent assay. Tubercle and lung disease. 1993; 74: 200-203.

20 Gupta, S., Bhatia, K. and Dalta, K.K. Scrological diagnosis of childhood tuberculosis by estimation of Mycobacterium antigen 60 specific imunogloblulins in serum. Tubercle and lung diseaes. 1997; 78:21-27.

21. Roos, B.R., Van, Cleef, M.R., Githui. et al. Cost effectiveness of the PCR versus smear examination for the Diagnosis of tuberculosis in Kenya. a theoretical model. Intern. $J$ tuberculosis and lung disease. 1998; 2(3): 235-241.

22. Gocim, A., Kiper N, Erstan U., Kalyai, O. and Ozcelik, $\mathrm{U}$. Is the BCG test of diagnostic value inTB? Tubercle and lung disease 1994; 75:54-57.

23 Ndugwa, C.M., Nduati, R.W. and Bhat G.J. HIV and AIDS in children. Primary Health Care Manual. Editors: Kopano M. Onyango F, Bwibo N. UNICEF pp 1995; 110-121.

24. Ellard, G.A. and Fourie, P.B. Rifampicin bioavailability and the therapeutic necessity for ensuring Absorption (review). Intern. J. Tuberculosis and Lung Dis. 1999; 3(11 suppl 3): $317-321$

25. Chakaya, J.M., Kibuga D., Nganga, L., et al. Tuberculosis re-treatment outcomes within the public service in Nairobi, Kenya. East Afr. Med. J. 2002; 79:11-15. 